\title{
ANALYSIS TURN-TAKING USED BY PRINCESS POPPY AND BRANCH IN THE TROLLS MOVIE
}

\author{
Iis Karmila1, Ida Lisdawati ${ }^{2}$ \\ ${ }^{1}$ IKIP Siliwangi \\ ${ }^{2}$ IKIP Siliwangi \\ ${ }^{1}$ iiskarmila97@gmail.com, ${ }^{2}$ idalisdawati9088@gmail.com
}

\begin{abstract}
This research about spoken language which is discussed in discourse analysis. In this research, researcher only focused on turn-taking, especially in the overlap and interruption section. Reseacher used quantitative methods and Guttman scale in processing data obtained from conversation in a movie called Trolls. Researcher choose a movie because in general a movie always contains conversations as well as in that movie. In the movie, researcher only analyzed 2 characters, Princess Poppy and Branch. The purpose of this research was to determine how much turn-taking was used by Princess Poppy and Branch. The results of the analysis showed that the turn-taking by Princess Poppy was 157 while the Branch was 110. The researcher used a table to show the results based on the theory of turn-taking. We can see in table 1 and table 2 that the amount of overlap is more dominant than interruption. The result of the analysis showed frequency overlap Princess Poppy is $82 \%$ of the total 128 and interruption frequncy is $18 \%$ of the total 29 times. While in tabel 2 it shows that the number of overlap conducted by Branch was 81 with a frequency of $74 \%$ and 29 interruption with a frequency of $26 \%$.
\end{abstract}

Keywords: Discourse Analysis, Interruption, Overlap, Spoken Language, Turn-taking

\section{INTRODUCTION}

Language is a tool for communication. In communication there must be a conversation between people who carry out the interaction. In a conversation there is namely turn-taking. Turn-taking is kind of spoken language discussed in discourse analysis. Discourse analysis is a research method for studying a language whether written or spoken depending on its social context. It aims to understand how language is used in real life situations. In other line, discourse analysis relates to the context and languge in which and how it is used in the books by Toolan and McCarthy (2006) as cited in (Setiawan, Ningtyas, \& Lisdawati, 2019). In other words, discourse is often translated as serious writing or conversation, rational and neatly arranged in the organization (Syam, 2019). Thus, discourse can take the form of poetry, essays, speeches or diologues. Based on definition above discourse analysis is used to study language in social context. It focuses on the purposes and effects of written and spoken communivation. In discourse analysis there are several sub-chapters, one of which is spoken language.

In language there are to ways to communicate, such as writing and spoken language. Spoken language is the language used by sound. Generally, spoken language is better understood in the importance of dialogue (Bothe, Magg, Weber, \& Wermter, 2018). There are several kind in spoken language such as; adjacency pairs, turn-taking, repairs, sequences, feedback, opening and closing. But, in this research, researcher only focus on turn-taking. Turn-taking is the transition to talk in a conversation. Turn-taking comprises how the speakers change and manage their turn in conversation. In other line, involves at least three strategies: taking the turn, holding the turn and yielding to the turn Strenstrom (1994) as cited in (Natalia, Subekti, \& Mirahayuni, 
2019). While, there is four in turn-taking sub-stategies such as starting up, taking over, interruption and overlap (Natalia et al., 2019). In this research, the reseacher intend to analyze interruption and overlap.

Interruption can be reffered to as acts that show classification, repetition, asking for help, rejection and resolution. Nevertheless, interruption is a common phenomenon in verbal conversations and it occurs when a speaker violates the other speaker's turn (Adeeb \& Abbas, 2019). In other line, interruption is a linguistic strategy that interferes with someone or robs them of trying to obstruct the rights of others to speak (Sapabsri, Dhanesschaiyakupta, Ackrapong, \& Phimswat, 2018). Furthermore, interruption is the speaker one starts talking before the other speaker finishes speaking while overlap is a turn when one speaker starts overlapping to take control of the conversation (Weatherall \& Edmonds, 2018). From the definition above interruption is a common phenomenom happens is casual talk that usually goes on briefly. However, many people think interruption is disturbing and it should be avoided in order to be establish a proper conversation. An overlap happens when the next speaker starts speaking at the time when the currant speaker almost finishes their utterance.

Turn-taking is often used in a conversation in the form of talkshow or movie. However, researcher choos the conversation in a movie to analyze the turn-taking used. The title of the movie is trolls, where the main character is princess Poppy in which voice actor is Anna Kendrick and Branch voice actor is Jastin Timberlake. Trolls movie is a 2016 American computer-animated musical comedy, created by Thomas Dam and produced by Gina Shay.

\section{METHOD}

Quantitative research method have been very common procedures for conducting research. According to Zein et al. (2019) quantitative is an approach that is used as a method in research by producing output in a form of statistical figures. This research will be conducted using a quantitative method to collecting data as the objectives. In collecting data for this research, researcher produces data by princess Poppy and Branch in the trolls movie. In this research, researcher choose trolls movie because even though the movie is in the form of animation, but it can not be denied that movie can attract the attention of all people. Besides being dominated by singing, the movie also contains conversations which of course use turn-taking. Because basically a conversation is carried out by two or more people so there is a turn-taking.

The researcher use several steps and Guttman scale to collecting the data. Firs read the theory about spoken language. Second, choose one of the part in spoken language. Third, the researcher choose the media that will research, such as movie, novel, speech etc and the reseacher choose the movie that will be analysis is Trolls. Forth, search the script to help researcher in analysis and journal the related with the theory. Finally, find the data and make a conclusion.

\section{RESULTS AND DISCUSSION}

\section{Results}

In this analysis researcher used the data from dialogue in the movie. After watching and understanding of that movie the researcher got the result based on the turn-taking theory.

Table 1. Turn-taking used by Princess Poppy in the Trolls movie 


\begin{tabular}{|c|c|c|c|}
\hline No. & Time & Dialogue Overlap & Dialogue Interruption \\
\hline 1. & 07.55 & Harmony & \\
\hline 2. & 08.01 & Yep. & \\
\hline 3. & 08.04 & $\begin{array}{l}\text { So do I. But that wouldn't leave } \\
\text { much time. }\end{array}$ & \\
\hline 4. & 08.11 & $\begin{array}{l}\text { You bet! But just because it's the } \\
\text { only way they'll ever be happy. }\end{array}$ & \\
\hline 5. & 08.31 & $\begin{array}{l}\text { Don't worry. No Troll ever will be } \\
\text { and that's why we're celebrating } \\
\text { with the biggest party ever. } \\
\text { Everybody's gonna be there. }\end{array}$ & \\
\hline 6. & 08.43 & Everybody & \\
\hline 7. & 10.52 & $\begin{array}{l}\text { Good. I was worried we weren't } \\
\text { projecting enough. }\end{array}$ & \\
\hline 8. & 11.16 & They're not gonna find us. & \\
\hline 9. & 11.26 & $\begin{array}{l}\text { You mean you're not coming to the } \\
\text { party tonight? }\end{array}$ & \\
\hline 10. & 11.44 & $\begin{array}{l}\text { Yes. I think everyone deserves to } \\
\text { be happy. }\end{array}$ & \\
\hline 11. & 11.49 & $\begin{array}{l}\text { Branch, I know you have happiness } \\
\text { inside you. You just need our help } \\
\text { to find it. }\end{array}$ & \\
\hline 12. & 12.08 & What do you say Branch? & \\
\hline 13. & 12.49 & & Hug time! \\
\hline 14. & 13.24 & I guess. & \\
\hline 15 . & 14.49 & $\begin{array}{l}\text { Okay, everyone. I just want to take } \\
\text { a moment... and get a little real. }\end{array}$ & \\
\hline 16. & 14.59 & & $\begin{array}{l}\text { I'd like to take a second to } \\
\text { celebrate our king... My } \\
\text { father... saved all of us from } \\
\text { those dreaded... }\end{array}$ \\
\hline 17. & 15.30 & Cupcake? Run! Run! Run! & \\
\hline 18. & 16.02 & Hurry! & \\
\hline 19. & 17.45 & & We have to rescue them. \\
\hline 20. & 17.51 & & $\begin{array}{l}\text { What about "No Terpll left } \\
\text { behind" }\end{array}$ \\
\hline 21. & 18.02 & Then I'll go. I'll go and save them & \\
\hline 22. & 18.08 & I have to at least try. & \\
\hline 23. & 18.45 & $\begin{array}{l}\text { Branch! Branch! Branch! Branch, } \\
\text { are you in there? Huh? }\end{array}$ & \\
\hline 24. & 18.51 & $\begin{array}{l}\text { The Party's over. We just got } \\
\text { attacked by a bergen. }\end{array}$ & \\
\hline 25. & 19.06 & Branch! & \\
\hline 26. & 19.08 & I have to tell you something. & \\
\hline 27. & 19.11 & I was just gonna... & \\
\hline 28. & 19.18 & The bergen's gone! & \\
\hline 29. & 19.26 & $\begin{array}{l}\text { No. It left! It took Cooper, and } \\
\text { Smidge, and Fuzzbert... and Satin, }\end{array}$ & \\
\hline
\end{tabular}




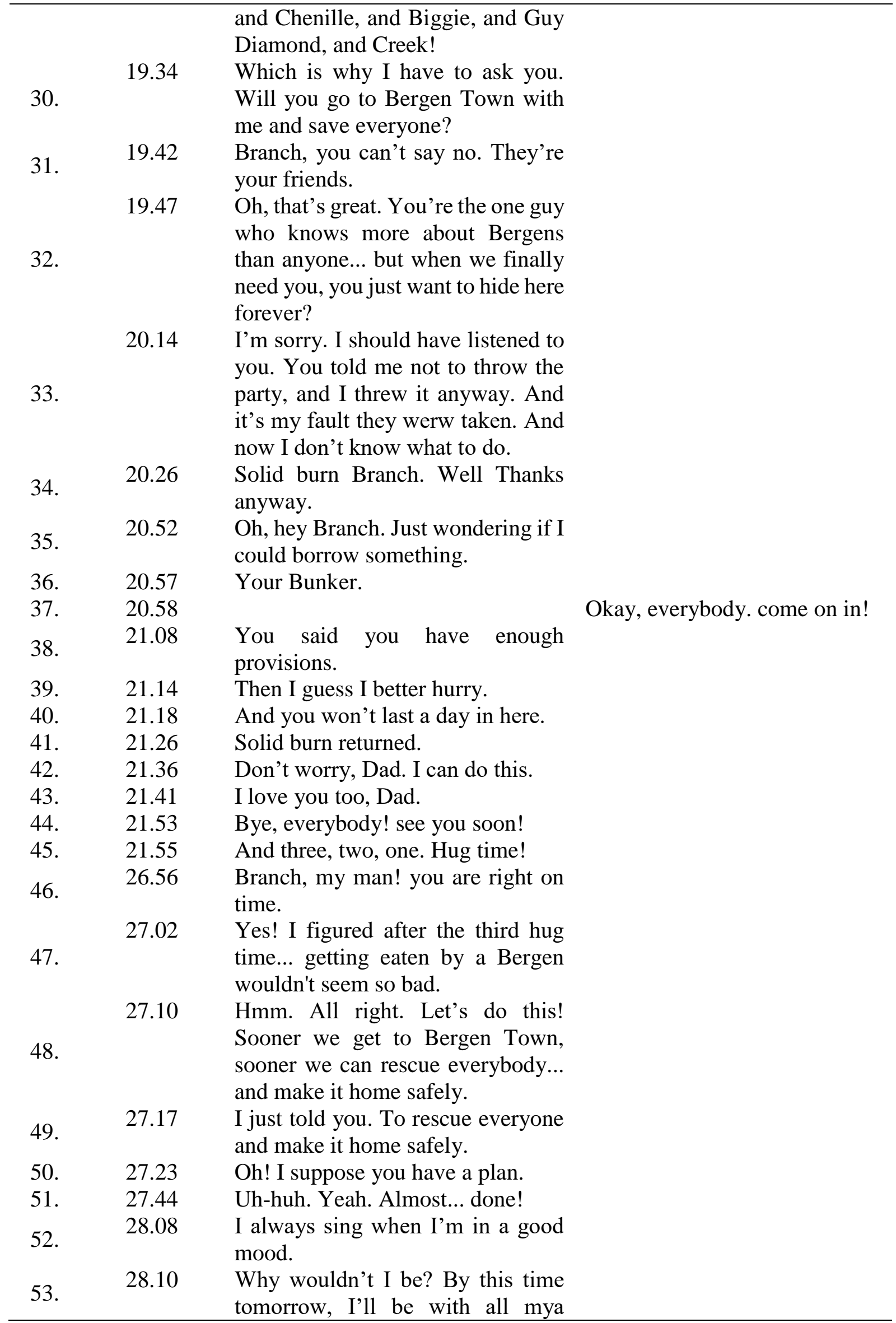


friends! Oh,I wonder what they're doing right now.

54. $28.17 \quad$ They're alive, Branch. I know it! 28.31

55.

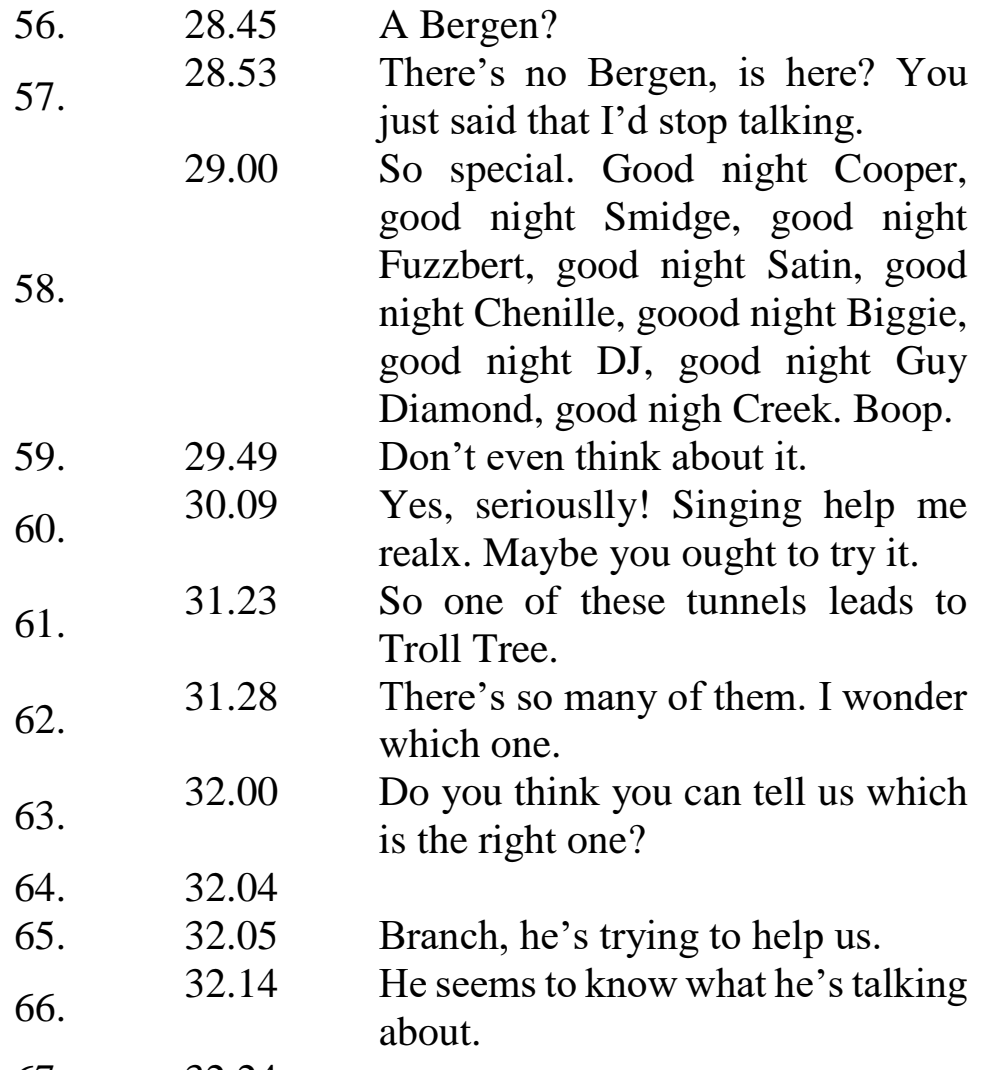

67. $\quad 32.24$

32.48

68.

69. $33.08 \quad$ Classic.

$70 . \quad 32.12$

71. $\quad 33.20$

$72 . \quad 33.50$

$73 . \quad 34.05$

34.55

74.

$75 . \quad 38.48$

He's just a cloud!

Branch! He can help us! Come back! Run, Cloud Guy!

The troll tree.

Wow! They're as miserable as you.

Which means they haven't eaten a Troll yet. Now, come on. Let's go save our friends.

So where do you think our friends
Hey, I know it's not all cupcakes and rainbows. But I'd rather go through life thinking that is mostly is... instead of being like you. You don't sing, you don't dance. So grey all the time! what happened to you?

Great!

Oh, I love high fives. I'll do it. Branch, it's a high five. The others lead to certain death. Get perspective.

Wait! are? 


\begin{tabular}{|c|c|c|c|}
\hline 76. & 38.54 & $\begin{array}{l}\text { Could you try to be positive? Just } \\
\text { once. You might like it. }\end{array}$ & \\
\hline 77. & 39.03 & $\begin{array}{l}\text { Thank you. That wasn't so hard, } \\
\text { was it? Branch! }\end{array}$ & \\
\hline 78. & 39.10 & & Listen. \\
\hline 79. & 39.30 & Branch, look! & \\
\hline 80. & 39.33 & $\begin{array}{l}\text { And on a silver platter, too. We } \\
\text { were both right. }\end{array}$ & \\
\hline 81. & 40.19 & & Creek! \\
\hline 82. & 41.10 & & No! \\
\hline 83. & 41.28 & Branch, we have to save him! & \\
\hline 84. & 41.31 & $\begin{array}{l}\text { We didn't see him crew. We didn't } \\
\text { see him swallow! }\end{array}$ & \\
\hline 85. & 44.07 & Oh, she's in love with the king. & \\
\hline 86. & 44.11 & $\begin{array}{l}\text { Well, maybe you don't know } \\
\text { everything about the Bergens. now } \\
\text { let's go. }\end{array}$ & \\
\hline 87. & 44.22 & Guys! & \\
\hline 88. & 44.41 & The sooner we can save creek! & \\
\hline 89. & 45.05 & & $\begin{array}{l}\text { I don't think he's alive. I hope } \\
\text { he's alive, and that's enough. }\end{array}$ \\
\hline 90. & 45.14 & & There's always a bright side. \\
\hline 91. & 45.34 & & $\begin{array}{l}\text { Bridget, stop! you're in love } \\
\text { with king Gristle. }\end{array}$ \\
\hline 92. & 45.53 & $\begin{array}{l}\text { Bridget, I can help you! What if } \\
\text { there was a way, we could both get } \\
\text { what we want? }\end{array}$ & \\
\hline 93. & 46.10 & $\begin{array}{l}\text { No Bridget, no. That Troll king } \\
\text { Gristle put in his mouth, that's } \\
\text { Creek. And I would do anything to } \\
\text { save him. The only problem is... we } \\
\text { can't get anywhere near theking } \\
\text { without him eating us. Oh, but... } \\
\text { you can. You can walk right up to } \\
\text { him and tell him how you feel. }\end{array}$ & \\
\hline 94. & 46.42 & $\begin{array}{l}\text { What if he didn't know you were a } \\
\text { scullery maid? What if he thought } \\
\text { you were his total babe? }\end{array}$ & \\
\hline 95. & 47.06 & We can help with that too! & \\
\hline 96. & 47.09 & $\begin{array}{l}\text { What do you say, Bridget? You } \\
\text { geet us Creek, and we'll get you a } \\
\text { date with the king. }\end{array}$ & \\
\hline 97. & 47.38 & & Branch! \\
\hline 98. & 48.01 & $\begin{array}{l}\text { Branch, what are you doing? You } \\
\text { have to sing! }\end{array}$ & \\
\hline 99. & 48.05 & Well, you have to! & \\
\hline 100. & 48.07 & No, you can. You just won't. & \\
\hline 101. & 48.09 & You have to! & \\
\hline 102. & 48.10 & Yes! & \\
\hline
\end{tabular}




\begin{tabular}{|c|c|c|c|}
\hline 103. & 48.11 & Why not? Why won't you sing? & \\
\hline 104. & 48.29 & $\begin{array}{l}\text { How did singing kill your } \\
\text { grandma? What song was she } \\
\text { singing? }\end{array}$ & \\
\hline 105. & 49.40 & $\begin{array}{l}\text { I'm so sorry, Branch. I had no idea. } \\
\text { I just assumed you had a terrible } \\
\text { voice. }\end{array}$ & \\
\hline 106. & 50.03 & I just thought you could use one. & \\
\hline 107. & 50.19 & Okay, people. Hair we go! & \\
\hline 108. & 51.41 & & And so are you. \\
\hline 109. & 51.45 & & $\begin{array}{l}\text { No, no, no! I got to get out of } \\
\text { here. I'll be right here for you, } \\
\text { Bridget. we all will. You'll twll } \\
\text { me what to say, right? }\end{array}$ \\
\hline 110. & 51.55 & Just wait until weget inside. & \\
\hline 111. & 53.01 & $\begin{array}{l}\text { We have to warm him up first. } \\
\text { Don't you know anything about } \\
\text { romance? }\end{array}$ & \\
\hline 112. & 53.06 & Really? & \\
\hline 113. & 53.46 & & Bridget! compliment him back! \\
\hline 114. & 53.49 & & $\begin{array}{l}\text { No! I meant say anything nice } \\
\text { about him. }\end{array}$ \\
\hline 115. & 58.32 & $\begin{array}{l}\text { Oh, I could scream too! Creek is } \\
\text { alive! Yay! }\end{array}$ & \\
\hline 116. & 58.45 & Branch, what's wrong? & \\
\hline 117. & 58.51 & $\begin{array}{l}\text { Well, you're gonna have plenty of } \\
\text { practice, because we're gonna save } \\
\text { Creek... and life will be all } \\
\text { cupcakes and rainbowa again. }\end{array}$ & \\
\hline 118. & 59.00 & $\begin{array}{l}\text { Okay, everybody... Let's go save } \\
\text { Creek. }\end{array}$ & \\
\hline 119. & 59.17 & $\begin{array}{l}\text { You don't wanna pretend to be } \\
\text { awesome you're not forever! }\end{array}$ & \\
\hline 120. & 59.23 & $\begin{array}{l}\text { Bridget, you don't need us } \\
\text { anymore. }\end{array}$ & \\
\hline 121. & 59.36 & Bridget... & \\
\hline 122. & 59.40 & Please, listen. Bridget! & \\
\hline 123. & 1.00 .25 & There it is! & \\
\hline 124. & 1.00 .37 & $\begin{array}{l}\text { Creek, we'll have you out of there } \\
\text { in a second. Hurry! it's stuck! }\end{array}$ & \\
\hline 125. & 1.0042 & Run! & \\
\hline 126. & 1.02 .10 & No! He can't be gone. & \\
\hline 127. & 1.02 .36 & & $\begin{array}{l}\text { You'll never find them. Not } \\
\text { where they're hiding. }\end{array}$ \\
\hline 128. & 1.02 .54 & Creek! you're alive! & \\
\hline 129. & 1.02 .59 & & $\begin{array}{l}\text { Branch! Wait! I'm sure there's } \\
\text { a reasonable explanation. At } \\
\text { least give him a chance. }\end{array}$ \\
\hline 130. & 1.03 .15 & You better explain yourself, Creek! & \\
\hline
\end{tabular}




\begin{tabular}{|c|c|c|}
\hline 131. & 1.03 .46 & \\
\hline 132. & 1.05 .30 & $\begin{array}{l}\text { I'm doing great. i got everybody I } \\
\text { love thrown in a pot. Thanks for } \\
\text { asking. }\end{array}$ \\
\hline 133. & 1.05 .42 & Yes! \\
\hline 134. & 1.05 .47 & $\begin{array}{l}\text { I'm sorry. I don't know why I } \\
\text { thought I could save you. All I } \\
\text { wanted to do was keep everyone } \\
\text { safe, like you did, Dad. But I } \\
\text { couldn't. }\end{array}$ \\
\hline 135. & $\begin{array}{l}1.06 .04 \\
1.06 .12\end{array}$ & I let everyone down. \\
\hline \multicolumn{3}{|l|}{136.} \\
\hline 137. & 1.11 .51 & Thank you! \\
\hline 138. & 1.11 .55 & For waht? \\
\hline 139. & 1.11 .59 & $\begin{array}{l}\text { Really? You're finally happy? } \\
\text { Now? }\end{array}$ \\
\hline 140. & 1.12 .17 & $\begin{array}{l}\text { I don't know. But I know we're } \\
\text { noot giving up. }\end{array}$ \\
\hline 141. & 1.12 .38 & Bridget? \\
\hline 142. & 1.12 .42 & What are you doing? \\
\hline 143. & $\begin{array}{l}1.12 .43 \\
1.12 .50\end{array}$ & But... \\
\hline
\end{tabular}

145. $1.12 .54 \quad$ But, Bridget...

146. 1.13.13 I love you too, Bridget.

147. 1.13.22 Come with us.

148. 1.13.28 Bridget!

149. 1.14.47 Come on. Everybody, let's go. No Troll left behind!

1.15.03 Bridget just ruined her life to save

150. ours. It's not right! She deserves to be happy as much as we do. They all do!

151.

$$
1.16 .38
$$

152.

153. 1.17.03 That feeling? That was happiness

154.

$$
1.17 .16
$$

1.17 .52

155.
No! Creek, please don't do this.

You were right, Branch. The world isn't all cupcakes and rainbows

No! Bridget, if you gp in there without us, you know what they'll do.

Beacuse she didn't think you would want awesome like her. Wait! king Gristle, when you were with Bridget, you were feeling something, weren't you?

But king Gristle's never eaten a Troll in his life, right?

No! happiness isn't something you put inside. It's already there. Sometimes you just need someone to help you find it. 


\begin{tabular}{lll}
\hline 156. & 1.18 .11 & $\begin{array}{l}\text { Of course! It's inside you! It's } \\
\text { inside of all of us! And I don't think } \\
\text { it. i feel it! } \\
\text { Now that I am queen, I decree that } \\
\text { hug time is all the time. }\end{array}$ \\
\hline Total & 1.21 .18 & \multicolumn{1}{c}{128} \\
\hline
\end{tabular}

Table 2. Turn-taking used by Branch in the Trolls movie

\begin{tabular}{|c|c|c|}
\hline No. & Time & Dialogue Overlap \\
\hline 1. & 10.46 & $\begin{array}{l}\text { Unbelievable, guys. Really. really } \\
\text { grat. Gopd job. I could hear you } \\
\text { from a mile away! }\end{array}$ \\
\hline 2. & 10.55 & \\
\hline 3. & 11.06 & $\begin{array}{l}\text { No, I don't. The Bergens are } \\
\text { coming! The Bergens are coming! } \\
\text { The Bergens are coming! }\end{array}$ \\
\hline 4. & 11.20 & $\begin{array}{l}\text { No, they're not going to find me, } \\
\text { because I will be in my highly } \\
\text { camouflaged... heavily fortified, } \\
\text { Bergen-proof survival bunker. }\end{array}$ \\
\hline 5. & 11.35 & $\begin{array}{l}\text { Bi? Load? Crazy? You're just } \\
\text { gonna lead the Bergens right to us! }\end{array}$ \\
\hline 6. & 11.47 & I don't do happy. \\
\hline 7. & 12.14 & $\begin{array}{l}\text { I wouldn't be caught dead at your } \\
\text { party, but you will be. Caught and } \\
\text { dead. }\end{array}$ \\
\hline 8. & 12.41 & $\begin{array}{l}\text { Okay, fine. I'm positive you all are } \\
\text { going to get eaten. }\end{array}$ \\
\hline
\end{tabular}

9.

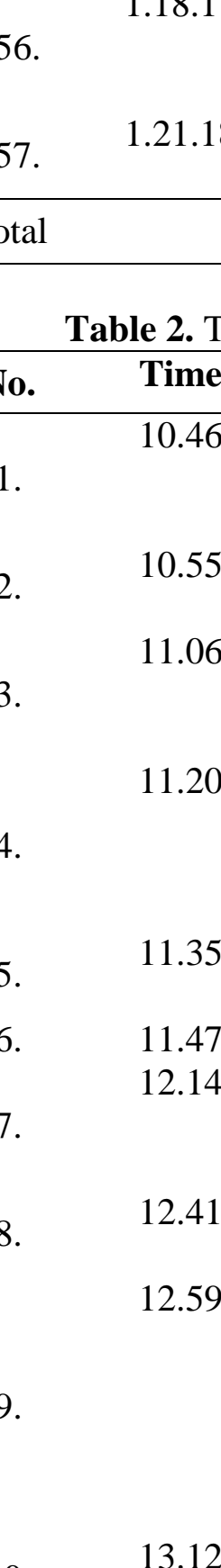

Poppy, if I can hear you,so can the Bergens.

Dialogue Interruption

Sens

Someday, when the Bergens find us... and the survival of every Troll is in your hands... I sure hope the answer is singing, dancing and huging... beacuse that's all you know how to do.

10. $\quad 13.12$ I can't believe you're gonna be queen one day.

11. $\quad 18.49 \quad$ I'm not going to your party.

12. 18.53 I knew it.

$13 . \quad 19.07$

14. $\quad 19.09$

19.12

Ssshhhh

Ssshhhh

Sshh! Sshh! What? What could

15. be so important thatit's worth leading the Bergen right to us?

19.20 You don't know that. I could still be

16. out there. Watching. Waiting. Listening.

17. $19.34 \quad \mathrm{Mmm}$ 


\begin{tabular}{|c|c|c|c|}
\hline 18. & $\begin{array}{l}19.40 \\
19.44\end{array}$ & What? No. & $\begin{array}{l}\text { Uh-uh-uh. They're your } \\
\text { friends. I'm staying right here } \\
\text { in my bunker where it's safe. }\end{array}$ \\
\hline 20. & 19.53 & $\begin{array}{l}\text { Forever? Pfft. No. I really only } \\
\text { have enough supplies which I am. } \\
\text { You all said I was crazy, huh? Well, } \\
\text { who's crazy now? Me? }\end{array}$ & \\
\hline 21. & 19.56 & Crazy prepared. & \\
\hline 22. & 20.23 & $\begin{array}{l}\text { Why don't you try csrapbooking } \\
\text { them to freedom? }\end{array}$ & \\
\hline 23. & 20.32 & & Oh, hey. \\
\hline 24. & 20.56 & What? & \\
\hline 25 . & 20.58 & What? & \\
\hline 26. & 21.03 & & $\begin{array}{l}\text { No! No! No! Whoa, wait! } \\
\text { poppy, what are you doing? }\end{array}$ \\
\hline 27. & 21.10 & & It'll last them two wwks! \\
\hline 28. & 21.16 & & $\begin{array}{l}\text { Wait, wait, wait! You won't last } \\
\text { a day out there. }\end{array}$ \\
\hline 29. & 22.07 & & No. \\
\hline 30. & 22.08 & & No! No! \\
\hline 31. & 27.00 & $\begin{array}{l}\text { Oh, right. like you knew I was } \\
\text { coming. }\end{array}$ & \\
\hline 32. & 27.05 & $\begin{array}{l}\text { And I figured there was no way you } \\
\text { could do this by yourself. Guess we } \\
\text { were both right. }\end{array}$ & \\
\hline 33. & 27.15 & & $\begin{array}{l}\text { Wait, wait, wait. What's your } \\
\text { plan? }\end{array}$ \\
\hline 34. & 27.20 & $\begin{array}{l}\text { Okay. That's not a plan. That's a } \\
\text { wish list. }\end{array}$ & \\
\hline 35. & 27.26 & $\begin{array}{l}\text { First.... we get to the edge of } \\
\text { Bergen Town without being } \\
\text { spotted. Then, we get inside by } \\
\text { sneakinng through the old escape } \\
\text { tunnels... which will then lead us to } \\
\text { the Troll Tree.... right before we get } \\
\text { caught, and suffer a miserable } \\
\text { death... at the hands of horrible, } \\
\text { bloodthirsty Bergen! Hold on a } \\
\text { second, are you scrapbooking my } \\
\text { plan? }\end{array}$ & \\
\hline 36. & 27.57 & $\begin{array}{l}\text { There will be no more... } \\
\text { scrapbooking. }\end{array}$ & \\
\hline 37. & 28.06 & Do you have to sing? & \\
\hline 38. & 28.09 & Do you have to be in a good mood? & \\
\hline 39. & 28.16 & Probably being digested. & \\
\hline 40. & 28.20 & $\begin{array}{l}\text { You don't know anything, Poppy. } \\
\text { And I can't wait to see the look on } \\
\text { your fae... when you realize the }\end{array}$ & \\
\hline
\end{tabular}




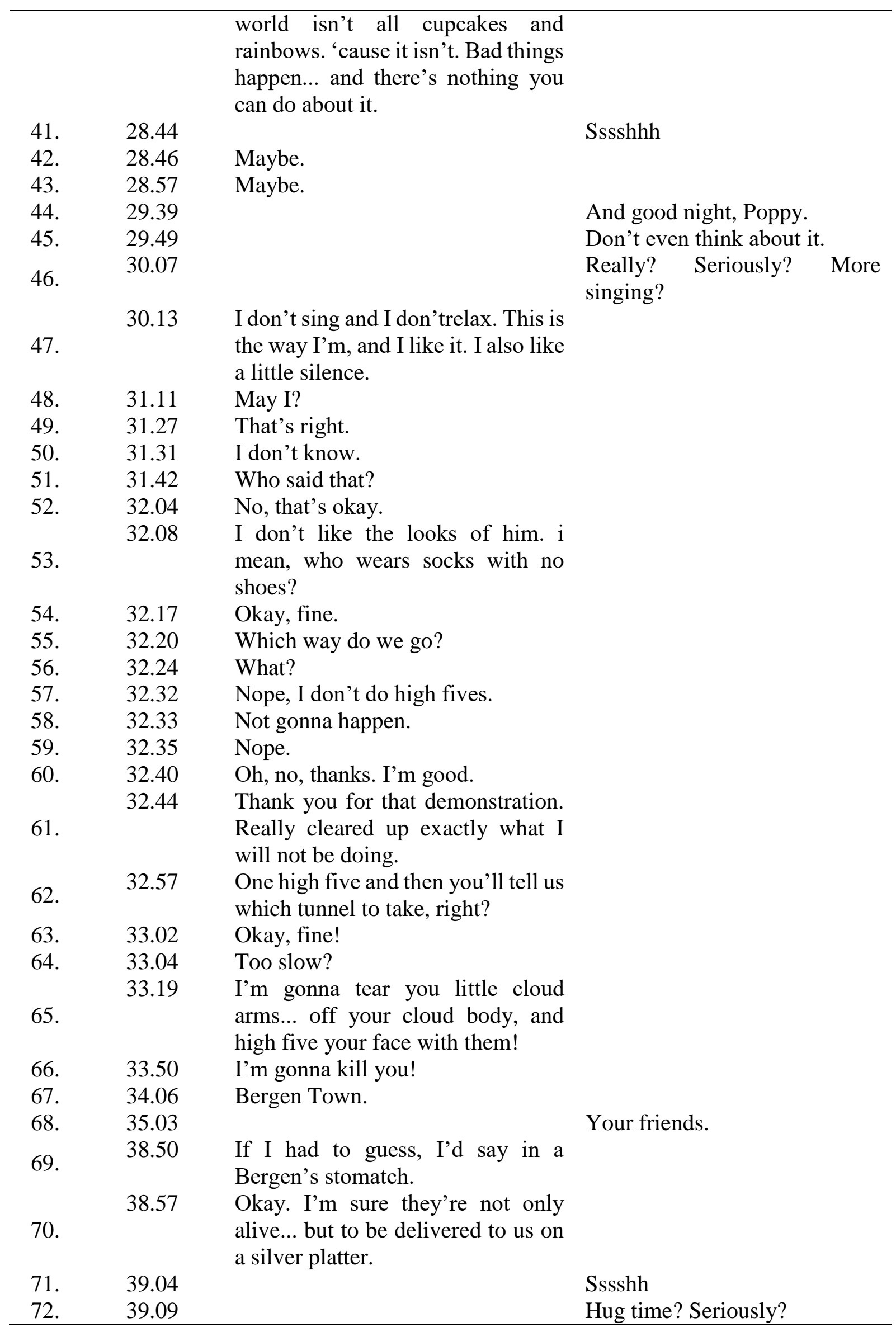




\begin{tabular}{ccl}
\hline 73. & 39.32 & $\begin{array}{l}\text { They're alive? } \\
\text { Save him from what? His } \\
\text { stomatch? }\end{array}$ \\
74. & 41.29 &
\end{tabular}

75.

Face it, Poppy. Sometimes people go into other people's mouths... and they don't come out. If we go after Creek now, we are going to get eaten. I'm sorry... but it's too late for him.

\begin{tabular}{|c|c|c|c|}
\hline 76. & 41.51 & Huh. poppy! & \\
\hline 77. & 44.08 & $\begin{array}{l}\text { What are you talking about? } \\
\text { Bergens don't have feelings. }\end{array}$ & \\
\hline 78 & 44.29 & & Ssshh \\
\hline 79. & 44.33 & & $\begin{array}{l}\text { No! There is not a } p \\
\text { on right here. The soo } \\
\text { you guys out of here. }\end{array}$ \\
\hline 80 . & 44.43 & & What? \\
\hline 81. & 44.51 & $\begin{array}{l}\text { I know you're looking for the } \\
\text { cupcakes and rainbows here... but } \\
\text { let's face it, Creek's been eaten. }\end{array}$ & \\
\hline 27 & 45.02 & $\begin{array}{l}\text { Poppy, how could you possibly } \\
\text { think Creek's still alive? }\end{array}$ & \\
\hline 83. & 45.10 & $\begin{array}{l}\text { How do you always look on the } \\
\text { bright side? There is no bright side } \\
\text { here. None! }\end{array}$ & \\
\hline 84. & 47.32 & & Oh, no. That's okay. \\
\hline 85. & 47.35 & I just don't sing. & \\
\hline 86. & 48.02 & I told you, i don't sing. & \\
\hline 87. & 48.06 & I'm sorry, i can't. & \\
\hline 88. & 48.08 & & Fine! I just won’t. \\
\hline 89. & 48.09 & & No! \\
\hline 90. & 48.12 & $\begin{array}{l}\text { Because singing killed my } \\
\text { grandma, okay? Now, leave me } \\
\text { alone. }\end{array}$ & \\
\hline 91. & 48.36 & I was the one singing. & \\
\hline 92. & 49.37 & I haven't sung a note since. & \\
\hline 93. & 49.48 & $\begin{array}{l}\text { No, no, it was like an angel's. At } \\
\text { least, that's what Grandma used to } \\
\text { say. Whoa, whoa. What are you } \\
\text { doing? It's not hug time. }\end{array}$ & \\
\hline 94. & 50.14 & $\begin{array}{l}\text { Okay. Okay, I'll help. But I'm still } \\
\text { not singing. }\end{array}$ & \\
\hline 95. & 52.59 & $\begin{array}{l}\text { When are you gonna ask him about } \\
\text { Creek? }\end{array}$ & \\
\hline 96. & 53.04 & Of course! i'm passionate about it. & \\
\hline 97 & 53.07 & $\begin{array}{l}\text { Don't you know anything about } \\
\text { sarcasm? }\end{array}$ & \\
\hline 08 & 53.55 & Poppy, help her. & \\
\hline
\end{tabular}




\begin{tabular}{|c|c|c|c|}
\hline 99. & 58.46 & $\begin{array}{l}\text { Nothing. I thoughtwe were } \\
\text { celebrating. }\end{array}$ & \\
\hline 100. & 58.50 & It's been a while. & \\
\hline 101. & 58.57 & Up top! Too slow. & \\
\hline 102. & 59.10 & & $\begin{array}{l}\text { The dinner where they're } \\
\text { serving Troll? Yeah, I think } \\
\text { we're gonna have to skip that } \\
\text { one. }\end{array}$ \\
\hline 103. & 1.02 .12 & I'm sorry, Poppy. We're too late. & \\
\hline 104. & 1.02 .57 & & He's selling us out! \\
\hline 105. & 1.06 .10 & But, Рoppy.... & \\
\hline 106. & 1.11 .52 & No. Thank you. & \\
\hline 107. & 1.11 .57 & For showing me how to be happy. & \\
\hline 108. & 1.12 .03 & $\begin{array}{l}\text { I think so. Happiness is inside of all } \\
\text { of us, right? Sometimes, you just } \\
\text { need someone to help you find it. }\end{array}$ & \\
\hline 109. & 1.15 .00 & Poppy? & \\
\hline 110. & 1.21 .14 & $\begin{array}{l}\text { I know it's not officially hug time } \\
\text { yet, but... }\end{array}$ & \\
\hline Total & & 81 & 29 \\
\hline
\end{tabular}

\section{Discussion}

In the movie titled Trolls has found 157 turn-takings used by Princess Poppy and 110 by Branch. To find the results data of the research, researcher used quantitative method with the Guttman scale frequency

Guttman scale $p=\frac{F}{n} \times 100 \%$

Explanation: $\mathrm{P}=$ Percentage, $\mathrm{F}=$ Frequency, $\mathrm{n}=$ Total, $100 \%=$ Constans. The turn-taking resulted in $82 \%$ overlap and $18 \%$ interruption used by Princess Poppy while the number committed by Branch was $74 \%$ overlap and $26 \%$ interruption.

\section{CONCLUSION}

In this research, the researcher concluded that each movie contains a conversation which in the conversation always uses turn-taking. So it becomes an interesting reason for researcher to conduct analyze turn-taking in a movie. It can be concluded that the total number of turn-taking undertaken by Poppy and Branch was 267. The turn-taking portion consisted of 209 overlaps and 58 interruptions

\section{ACKNOWLEDGMENTS}

Praise to Allah which gives researcher the opportunity to finish this article well and in good health. The researcher wants to thankful to All lecturers in English Education Study Program, especially for Mrs. Ida Lisdawati S.Pd, M.Hum. Not to be missed to say thank you to our family who always pray, support, and give motivation to the researcher to do the study at campus,and also the researcher want to thankful to all friends who support and help the researcher to finish the project. The researchers hope this research project can be helpful to the future reader and can be a references for other researcher. 


\section{REFERENCES}

Adeeb, E. R., \& Abbas, A. M. (2019). Sex- and Age-Based Approach to the Study of Interruption in "The Kings of Summer" Movie and "Pretty Little Liars" TV Series: A Case of Same-Sex Teenage Interactions. International Journal of English Linguistics, 9(2), 229. https://doi.org/10.5539/ijel.v9n2p229

Bothe, C., Magg, S., Weber, C., \& Wermter, S. (2018). Discourse-Wizard: Discovering Deep Discourse Structure in your Conversation with RNNs. Retrieved from http://arxiv.org/abs/1806.11420

Natalia, D. D., Subekti, F., \& Mirahayuni, N. K. (2019). 56 turn taking strategies in political debates. 2(2), 56-63.

Sapabsri, O., Dhanesschaiyakupta, U., Ackrapong, T. T., \& Phimswat, O.-U. (2018). an Analysis of Gender and Status Affecting Conversational Interruptions. PEOPLE: International Journal of Social Sciences, 4(1), 257-271. https://doi.org/10.20319/pijss.2018.41.257271

Setiawan, D., Ningtyas, Z. M., \& Lisdawati, I. (2019). Analyze Turn Taking in Talk Show About Education for Everyone on Channel Youtube. PROJECT (Professional Journal of English Education), 2(4), 489. https://doi.org/10.22460/project.v2i4.p489-493

Syam, A. T. (2019). The Analysis of System Constraints in National Treasure 2: Book of Secrets Movie. Language Circle: Journal of Language and Literature, 13(2). https://doi.org/10.15294/lc.v13i2.18968

Weatherall, A., \& Edmonds, D. M. (2018). Speakers formulating their talk as interruptive. Journal of Pragmatics, 123, 11-23. https://doi.org/10.1016/j.pragma.2017.11.008

Zein, S., Yasyifa, L., Ghozi, R., Haraphap, E., Badruzzaman, F., \& Darmawan, D. (2019). Pengolahan dan analisis data kuantitatif menggunakan aplikasi spss. 4, 1-7. 